\title{
The Relative Location of the Dye Staining Endpoint Indicated With Polypropylene Glycol-Based Caries Dye versus Conventional Propylene Glycol-Based Caries Dye
}

Daniel W Bostona

Steven R Jefferies ${ }^{b}$

John P Gaughan ${ }^{\circ}$

\section{ABSTRACT}

Objectives: This study determined the difference in the location of the caries dye staining endpoint of $1 \%$ Acid Red dye in propylene glycol versus that of $1 \%$ Acid Red dye in polypropylene glycol.

Methods: Freshly extracted permanent molar crowns with primary occlusal carious lesions were chisel-split axially to expose the lesion in cross-section on both halves. One half was stained with propylene glycol-based dye and the other with polypropylene glycol-based dye. For the control group, both halves were stained with propylene glycol-based dye. The dye staining front was marked on digital images of the stained split surfaces, and the images were aligned using reference notches. The distance between the marked staining front lines was measured in five locations, and the measurement protocol was repeated. Weighted averages and a $95 \%$ confidence interval for the distance between marked staining front lines were calculated for the control and experimental groups.

Results: The weighted average distance for the experimental group $10.298 \mathrm{~mm}, 95 \%$ confidence interval $0.240 \mathrm{~mm}-0.357 \mathrm{~mm}$ ) was about four times that of the control group $(0.070 \mathrm{~mm}, 95 \%$ confidence interval $0.051 \mathrm{~mm}-0.089 \mathrm{~mm}$ ). Generally, the marked staining line for the polypropylene glycol-based dye specimens was located shallow (occlusal) to the propylene glycol-based staining line (range $-0.12 \mathrm{~mm}$ to $0.66 \mathrm{~mm}$ ).

Conclusions: The staining endpoint of $1 \%$ Acid Red dye in polypropylene glycol is shallower than that of $1 \%$ Acid Red dye in propylene glycol. The method is useful for comparing staining endpoints of caries dye formulations. (Eur J Dent 2008;2:29-36)

Key words: Caries; Dye staining; Propylene glycol.

a Associate Professor and Chair, The Laura Carnell Professor of Restorative Dentistry, Department of Restorative Dentistry, Kornberg School of Dentistry, Temple University.

b Professor, Department of Restorative Dentistry, Kornberg School of Dentistry, Temple University. Associate Professor, Biostatistics Consulting Center, School of Medicine, Temple University.

- Corresponding Author: Daniel W. Boston, DMD Temple University, Kornberg School of Dentistry, Department of Restorative Dentistry, PA, USA.

E-mail: daniel.bostonatemple.edu

\section{INTRODUCTION}

The removal of infected carious dentin is an exacting procedure routinely accomplished during tooth preparation, prior to placing a restoration. Although explorer-verified hardness equal to that of normal dentin is traditionally considered the ideal endpoint of excavation, caries indicator dyes have been developed to provide a more objective and easily recognized visual differentiation for this endpoint. ${ }^{1-4}$ These dyes also distinguish between the outer layer of very soft highly infected 
carious dentin and the deeper layer of somewhat softened uninfected or at most lightly infected dentin. 5,6 Caries Detector ${ }^{\mathrm{TM}}$ (Kuraray America, NY, NY) and Caries Finder RedTM (Danville Materials, San Ramon, CAl are two representative products, consisting of $1 \%$ Acid Red 52 dye (sulforhodamine B) in propylene glycol. ${ }^{7}$

A recent approach to minimizing unwanted staining by caries indicator dyes has been to change the solvent for the dye compound. The rationale is based upon two studies that showed diminished diffusion into porous tissues of dyes dissolved in higher molecular weight solvents. ${ }^{8-10}$ Instead of propylene glycol (MW=76) (PG) or a mixture of PG and water as used in many currently marketed caries dye products, new formulations using larger molecules such as polypropylene glycol ( $M W=300$, for example) (PPG) have been proposed. In 2005, a US patent application was filed for this type of caries detecting solution. ${ }^{11}$ This application discloses a range of inventive examples including solutions containing various mixtures of PPG with a molar weight of 300 , polyethylene glycol with molar weights of 400 and 4000, PG, glycerin and water. Two new caries indicator dye products are now available in Japan (Caries Check Red ${ }^{\mathrm{TM}}$ and Caries Check Blue ${ }^{\mathrm{TM}}$, Nishika Co., Yamanashi, JPJ consisting of $1 \%$ Acid Red 52 or Brilliant Blue dye in 300 MW PPG.

A few studies do indicate, at least indirectly, that PPG-based dye solutions may provide a more conservative excavation endpoint. When the final excavated surfaces determined by PG-based dye solution or PPG-based dye solution were compared using micro-Vickers hardness (MVH), it was found that the PPG dye-determined group was significantly softer than the PG dye-determined group (mean MVH 32.7 vs. 44.7). ${ }^{12}$ Since hardness generally increases with increasing depth in dentin carious lesions this result would suggest a shallower, more conservative endpoint for the PPG dye-determined specimens. ${ }^{3}$ Because both groups were found to be free of bacteria histologically, it was concluded that the PPG dye-determined endpoint would be preferable. However, since hardness generally decreases with increasing depth in normal dentin, the clinical interpretation of hardness differences is somewhat obscured. After this initial report, studies incorporating laser fluorescence comparisons pointed toward a similar conclusion, at least for permanent teeth. The rationale for using laser fluorescence values ("DD") (Diagnodent, KaVo, USA) to assess differences in excavation endpoints was based upon a study of the spectral autofluorescence characteristics of cariouslesions, aswellas general guidelines for interpretation of DD values when detecting carious lesions in occlusal fissures. ${ }^{13-15}$ In extracted carious teeth, it was shown that the mean MVH was significantly lower and DD values significantly higher in the PPG dye-determined group versus the PG dye-determined group. ${ }^{16}$ In another study, a similar result was found for the group mean DD value in permanent teeth but not in primary teeth. ${ }^{17}$ Another study using extracted teeth containing acute or chronic carious lesions also found that the mean DD value was higher for the PPG dye-determined group versus the $P G$ dye-determined group. ${ }^{13}$ In a clinical study, this same result regarding DD values was confirmed for permanent teeth but not for primary teeth. ${ }^{8}$ One of these studies also found that all specimens first excavated to an endpoint indicated with the PPG-based dye could subsequently be re-stained with the PG-based dye, once again suggesting that PPG-based dye solutions may indicate a more conservative endpoint for excavation. ${ }^{13}$

More direct confirmation of this apparently more conservative endpoint indicated by PPGbased dyes is necessary for several reasons. First, although these initial studies do demonstrate a statistically significant difference in mean $\mathrm{MVH}$ and DD value between sample groups, it is not clear whether a difference exists in all carious lesions or just certain ones. In addition, although microhardness does generally increase with increasing depth in a dentin carious lesion, the relationship between microhardness and depth is neither linear nor constant from tooth to tooth. ${ }^{18}$ Similarly, although DD values do generally decrease with increasing depth in a dentin carious lesion, this inverse relationship is not linear and certainly not constant from tooth to tooth. ${ }^{19}$ This is true both at the unexcavated lesion surface and across the PG dye-guided excavation front. ${ }^{19}$ In light of these variables, and to assess clinical implications for cavity preparation, it is necessary to investigate the differences between the actual physical locations of surfaces created by PPG dyeguided excavation and those created by $P G$ dye- 
guided excavation.

The purpose of this study is to determine the difference in the location of the caries dye staining endpoint indicated by $1 \%$ Acid Red 52 dye in PG versus that indicated by $1 \%$ Acid Red 52 dye in PPG, in freshly extracted human permanent posterior teeth containing primary dentin carious lesions.

\section{MATERIALS AND METHODS}

Dye solutions were made by completely dissolving $0.100 \mathrm{~g}$ Acid Red 52 (sulforhodamine B, \#S-9012, Sigma Chemical Co., St. Louis, MO) in $10.00 \mathrm{ml}$ propylene glycol (Sigma \#P-1009) or in $10.00 \mathrm{ml}$ polypropylene glycol, (triol type, $\mathrm{MW}=300$ ) (\#160-17605, Wako Chemicals USA, Richmond, VAl at room temperature. Solutions were stirred by hand for ten minutes and vortex-mixed for two minutes twenty-four hours later. Dye solutions were stored in closed glass containers.

Freshly extracted, unrestored human permanent posterior teeth with primary occlusal carious lesions were collected under an IRBexempt protocol. Teeth were stored in sterile distilled water at $4^{\circ} \mathrm{C}$ for up to two weeks prior to processing. The outer surface of each specimen was cleaned with a soft-bristle toothbrush and sterile distilled water, sectioned horizontally at the dentino-enameljunction, the root portion discarded and the pulp tissue removed from the crown portion. Two opposing axial surfaces were ground flat, parallel to each other and perpendicular to the previous horizontal section plane using an 8" 240 grit carborundum disc mounted in a waterlubricated surface polisher (Model 900, Electron Microscopy Sciences, Hatfield, PA).

In preparation for splitting, a continuous fine groove was cut about $1 \mathrm{~mm}$ deep in the center of each ground axial surface running occlusogingivally and connecting across the occlusal surface approximately bisecting the external surface of the carious lesion. The groove was made with a 1" thin diamond disc mounted in a lowspeed handpiece. To provide identical reference marks on both split tooth halves, two to six orientation notches about $2 \mathrm{~mm}$ deep were made with a \#57 carbide bur across and perpendicular to the fine groove at various locations, but not in the region of the carious lesion. Figure 1 shows a specimen with notches and a groove, ready for splitting. The crown specimens were fractured along the groove using a 3/8" steel chisel and mallet yielding two segments, each containing a split surface showing the carious lesion in cross-section.

The fractured surfaces were then stained with caries dye. One of the two surfaces from each crown specimen, chosen at random, was stained with the PPG-based dye and the other with the PG-based dye. Two drops of dye were applied for ten seconds, rinsed under running tap water for ten seconds, the surface blotted damp-dry with a cotton gauze square and then observed under $2.6 x$ loupe magnification. Specimen pairs were discarded if they had not fractured along the fine groove or were missing tooth structure, if no lesion was observed in dentin or if the lesion extended to the pulp chamber, if the lesion was too dark to permit differentiation of the red dye staining, if the lesion was less than $1 \mathrm{~mm}$ into dentin, or if a previously unobserved restoration was detected. Of the 41 specimens originally collected, 18 were accepted for further analysis. As controls, three of the 18 fractured specimen pairs were stained on both surfaces with $1 \%$ sulforhodamine $B$ in $P G$. The other 15 specimen pairs were stained using both dyes, as described above. Each half-crown specimen was coded to "blind" which dye was used.

Color digital images were then made of each fracture surface. Using a digital microscope and camera (Optem Zoom-100, Qioptiq, Rochester, NY) (DP-11, Olympus America, Melville, NY) at 10x magnification and with ring light illumination

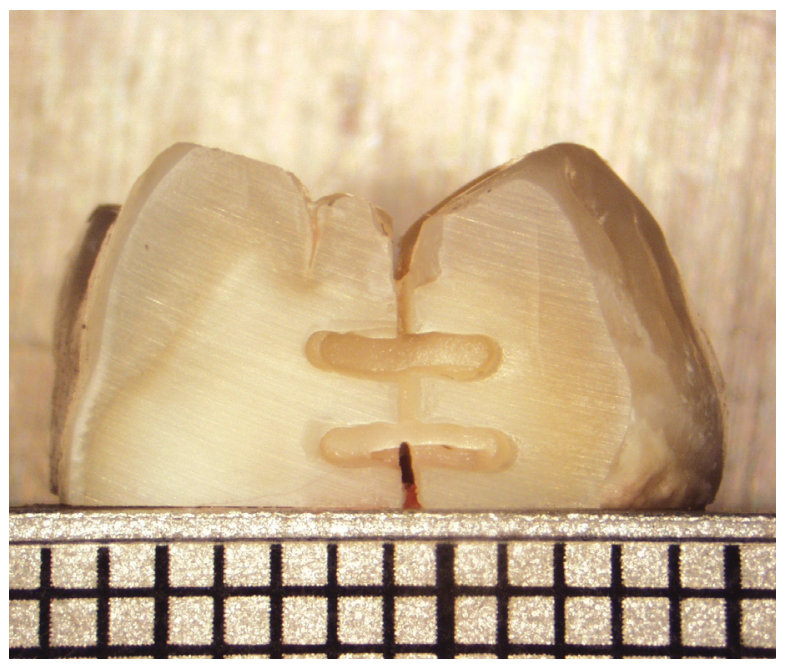

Figure 1. Specimen cut horizontally at the dentino-enamel junction to remove the roots, with one of two flattened axial surfaces visible, showing fine vertical groove to direct splitting, and two horizontal orientation notches. Grid lines represent $1 \mathrm{~mm}$. 
(\#EKE, Schott-Fostec, Auburn, NY), image files were created with the following settings: white balance $3000^{\circ} \mathrm{K}$, resolution set at $\mathrm{HQ} 1712 \times 1368$ pixels, ISO 100, ring light brightness 70 , and 1/15 sec exposure. Images were saved as .jpg files. To standardize the orientation of the fracture surface relative to the camera, both were mounted on a specimen stand (Olympus SZX-ILLK). A machinist square was fastened to the stand base to fix the position of a removable aluminum block on which specimens were mounted using a thin layer of boxing wax. For linear calibration, a millimeter grid was fixed to the block.

Image pairs were analyzed using a digital image analysis software program llmage Pro Discovery 4.5, Media Cybernetics, Silver Spring, MD). For each specimen, one of the two image pairs was opened, "flipped" left-to-right, and magnified 3x. Using a graphic tablet and digital pen (Intuos 3, Wacom, Vancouver, WA) the most pulpal extent of the red dye-staining was marked with a one pixel-wide yellow line. The other image of the pair was then opened, magnified $3 x$, and the pulpal extent of the dye-staining marked with a one pixel-wide blue line. The images were then combined and aligned. This was accomplished by selecting a rectangular "region of interest" on one image that included both the blue or yellow line as well as the millimeter reference grid, edge of the aluminum mounting block and reference notches in the specimen surface. The selected area was "copied" and "pasted" into the other image using a "50\% translucency" setting for the

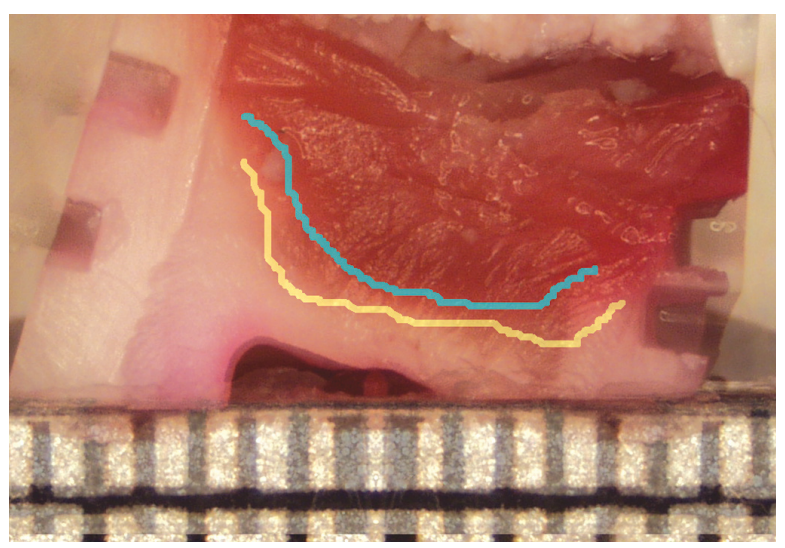

Figure 2. Combined image pairs from an experimental specimen showing the PG dye-staining boundary line (yellow) and the PPG dye-staining boundary line (blue). Lines have been redrawn and thickened to improve visibility for publication. The alignment notches can be seen on the left and right edges of the combined specimen images, and the aligned edges of the aluminum mounting block are visible. "paste" operation, providing a means to visually align the two image pairs on the reference notch edges and aluminum mounting block edge, while permitting both the blue and yellow marked lines to be clearly seen.

The distance between the yellow and blue lines was then measured using the software's linear measurement tool at 2x magnification after calibration based on the millimeter grid image. Distance was measured from line to line (L-L distance) at five locations beginning in the center of the lesion, with two additional measurements on either side, spaced $0.5 \mathrm{~mm}$ apart, perpendicular to the most occlusally located line and recorded in millimeters to two decimals. This measurement protocol was repeated once for all 15 experimental samples and for two of the three control samples, providing a total of 150 experimental (ten per sample) and 50 (five or ten per sample) control L-L distance measurements.

For each of the control (6) and experimental (15) specimen image pairs, mean $L-L$ distance values were calculated. Using these means, a weighted average was calculated for the combined control group and for the combined experimental group. The weighted averages were based on adjustment of the six or fifteen mean $L-L$ distance values from each specimen group by the variance of the individual $L-L$ distance values within that group. A 95\% confidence interval was calculated for the weighted averages.

To evaluate the test method, a 3-factor ANOVA (specimen, measurement, replication) on ranktransformed data was carried out on the control and experimental data from the individual specimens (control 6, experimental 15), using repeated measurements of each test pair (5), and replication of the measurement protocol (2). Prior to the ANOVA, the data in both the control and experimental groups were analyzed for normality using the Shapiro-Wilk test. All statistical analysis used SAS v9.1 software (SAS Institute, Cary, NC).

\section{RESULTS}

For 12 of the 15 experimental specimen image pairs, the line corresponding to PPG dye staining was located more occlusally (shallower) throughout its length than the line corresponding to $P G$ dye staining. For two image pairs, the PPG dye staining line was predominately located more 
occlusally, but there were areas where the two lines were coincident or where the $P G$ dye staining line was shallower. For these two specimen image pairs, this was true for both iterations of the image analysis protocol. In the specimen \#4 image pair, the $P G$ dye staining line was shallower for $12 \%$ (first iteration) and $51 \%$ (second iteration) of the line length, and coincident for $11 \%$ (first iteration onlyl. In the specimen \#8 image pair, the PG dye staining line was shallower for $14 \%$ and $19 \%$ of the line length and coincident for $5 \%$ and $13 \%$, respectively. For one specimen image pair (\#1), the dye staining lines were coincident for $2 \%$ of the line distance in one iteration only, and elsewhere the PPG dye staining line was shallower.
The measurement data for the control specimen image pairs is presented in Table 1. Mean L-L distances were small, ranging from $0.0377 \mathrm{~mm}$ to $0.0952 \mathrm{~mm}$. Individual distances ranged from $0 \mathrm{~mm}$ to $0.1910 \mathrm{~mm}$. Table 2 presents the measurement data for the experimental specimen image pairs. Mean $L-L$ distances were larger than for the controls, ranging from $0.153 \mathrm{~mm}$ to $0.506 \mathrm{~mm}$, while individual distances ranged from (-) $0.12 \mathrm{~mm}$ to (+) $0.66 \mathrm{~mm}$. Table 3 presents the data for the weighted averages of the means for the control and experimental specimen image pairs. For the control specimen image pairs, the weighted average $L-L$ distance was $0.70 \mathrm{~mm}$, with a $95 \%$ confidence interval of $0.51 \mathrm{~mm}$ to 0.89

Table 1. Data summary for the six control image pairs.

\begin{tabular}{|c|c|c|c|c|c|c|}
\hline Image \# & $\begin{array}{c}\mathrm{N} \\
\text { (measurements) }\end{array}$ & Mean (mm) & SD & Min & Max & Range \\
\hline 1 & 10 & 0.0747 & 0.0577 & 0 & 0.191 & 0.191 \\
\hline 2 & 10 & 0.0337 & 0.0293 & 0 & 0.088 & 0.088 \\
\hline 3 & 10 & 0.0745 & 0.0267 & 0.037 & 0.134 & 0.097 \\
\hline 4 & 10 & 0.0650 & 0.0352 & 0 & 0.123 & 0.123 \\
\hline 5 & 5 & 0.0952 & 0.0339 & 0.047 & 0.135 & 0.088 \\
\hline 6 & 5 & 0.0544 & 0.0277 & 0.030 & 0.100 & 0.070 \\
\hline
\end{tabular}

Table 2. Data summary for the fifteen experimental image pairs.

\begin{tabular}{|c|c|c|c|c|c|c|}
\hline Image \# & $\begin{array}{c}\mathrm{N} \\
\text { (measurements) }\end{array}$ & Mean (mm) & SD & Min & Max & Range \\
\hline 1 & 10 & 0.292 & 0.201 & 0.08 & 0.59 & 0.51 \\
\hline 2 & 10 & 0.196 & 0.110 & 0.04 & 0.38 & 0.34 \\
\hline 3 & 10 & 0.506 & 0.146 & 0.32 & 0.77 & 0.45 \\
\hline 4 & 10 & 0.165 & 0.175 & -0.12 & 0.44 & 0.56 \\
\hline 5 & 10 & 0.380 & 0.164 & 0.17 & 0.65 & 0.48 \\
\hline 6 & 10 & 0.180 & 0.121 & 0.02 & 0.43 & 0.41 \\
\hline 7 & 10 & 0.290 & 0.107 & 0.13 & 0.49 & 0.36 \\
\hline 8 & 10 & 0.270 & 0.135 & 0.04 & 0.47 & 0.43 \\
\hline 9 & 10 & 0.375 & 0.191 & 0.08 & 0.66 & 0.58 \\
\hline 10 & 10 & 0.153 & 0.051 & 0.07 & 0.22 & 0.15 \\
\hline 11 & 10 & 0.158 & 0.071 & 0.07 & 0.28 & 0.21 \\
\hline 12 & 10 & 0.185 & 0.075 & 0.05 & 0.32 & 0.27 \\
\hline 13 & 10 & 0.231 & 0.120 & 0.10 & 0.40 & 0.30 \\
\hline 14 & 10 & 0.193 & 0.075 & 0.07 & 0.29 & 0.22 \\
\hline 15 & 10 & 0.398 & 0.131 & 0.15 & 0.62 & 0.47 \\
\hline
\end{tabular}


$\mathrm{mm}$, versus a $0.298 \mathrm{~mm} \mathrm{~L}-\mathrm{L}$ distance and $95 \%$ confidence interval of $0.2140 \mathrm{~mm}$ to $0.357 \mathrm{~mm}$ for the experimental group. The weighted average $\mathrm{L}-\mathrm{L}$ distance for the experimental group was about four times that of the control group, with no overlap and $0.151 \mathrm{~mm}$ between the two confidence intervals.

The Shapiro-Wilk test for normality indicated that the control data was normally distributed $(P=.1226)$ but that the experimental data was not normally distributed ( $P=.0003)$. Therefore, ANOVA on ranks was used to detect effects by groups. At a significance level of $P=.05$, the ANOVA on ranks detected a significant effect by specimen and measurement, but not by replication in the control data. For the experimental data, an effect was detected by specimen only. Table 4 summarizes the ANOVA on Ranks results by effects for control and experimental groups.

\section{DISCUSSION}

The $0.298 \mathrm{~mm}$ weighted average $\mathrm{L}-\mathrm{L}$ distance for all experimental specimens is clinically relevant since it is possible to remove dentin in increments of this magnitude, potentially resulting in more conservative cavity preparations and even preventing unnecessary pulp exposures in some instances. This advantage is somewhat limited, however, because $0.3 \mathrm{~mm}$ represents perhaps one or two careful applications of a hand excavator or round bur, and because the $L-L$ distance ranged considerably: from $-0.12 \mathrm{~mm}$ to $0.66 \mathrm{~mm}$ within the specimens. With an aggressive excavation technique exceeding $0.3 \mathrm{~mm}$ or even less per increment, any advantage from a more conservative PPG-based caries dye endpoint would often be negated. However, in some cases, careful excavation using this PPG-based dye could actually prevent exposure in deep lesions approaching the pulp. Because carious lesions differ in type and amount of bacterial load, nutrient availability, and morphology and because dentin itself varies according location, age and reaction pattern, considerable variation would be expected in $L$ - $L$ distances among other specimen sets such as primary teeth, more chronic lesions, very active lesions, root lesions, and lesions associated with restorations.

The results of this study should be interpreted with caution, especially for clinical application. Since no difference was found in DD values for excavation surfaces created using PG-based versus PPG-based dyes in primary teeth in two previous studies, it may be that no $L-L$ distance difference exists in primary teeth. For this reason, the present study needs to be repeated for primary teeth. ${ }^{8,17}$ Other solvents or solvent mixtures as well as dyes other than sulforhodamine $B$ may produce different results, and would also need to be tested. The fractured dentin surface used in the present study does not include a smear layer as would

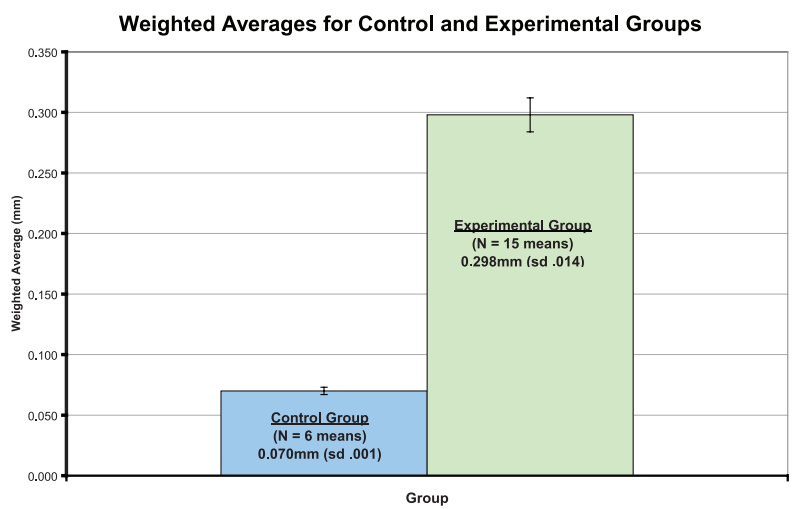

Table 3. Weighted averages data for control and experimental groups.

\begin{tabular}{|c|c|c|c|c|c|c|c|}
\hline Group & $\begin{array}{c}\text { Weighted } \\
\text { Average }(\mathrm{mm})\end{array}$ & $\mathrm{N}$ (means) & S.D. & Min & Max & Range & $\begin{array}{c}95 \% \\
\text { Confidence } \\
\text { Interval }\end{array}$ \\
\hline Control & 0.07 & 6 & 0.001 & 0.034 & 0.095 & 0.061 & $.051-.089$ \\
\hline Experimental & 0.298 & 15 & 0.014 & 0.153 & 0.506 & 0.353 & $.240-.357$ \\
\hline
\end{tabular}

Table 4. ANOVA on Ranks P-values by effects for control and experimental groups.

\begin{tabular}{lccc}
\hline Group & Specimen Effect & Measurement Effect & Replication Effect \\
\hline Control & .0172 & .0052 & .4044 \\
Experimental & $<.0001$ & .0510 & .7828 \\
\hline
\end{tabular}


sometimes be produced clinically by burs or hand excavators, and may, therefore, indicate a more conservative endpoint since a smear layer might stain with dye, having originated from elsewhere in the preparation. Therefore, the present study may represent a more idealized staining result compared to some clinical situations.

The differences in the weighted averages between the control and experimental groups as well as the results on the ANOVA on ranks analysis indicate that the present method is useful for differentiating staining endpoints between caries dyes. The method was able to detect a relatively small $L-L$ distance, approximately four times the average error seen in the control group which itself was very small. In both groups, a "specimen" effect was detected by ANOVA as would be expected for clinical specimens varying in lesion location, activity, size, etc. A "measurement" effect was detected in the control group and almost in the experimental group $(P=0.0510)$, also expected due to lesser variations in measurement orientation versus dentin tubule orientation, for example. A “repetitions" effect was not detected in either group, indicating an acceptable degree of consistency between runs. For future studies, errors might be reduced by more precise machining of the splitting groove and orientation notches, and by color-corrected thresholding of the stained specimen surface.

In the present study, any light pink staining encountered on the dye-stained fracture surface was treated as non-stained, and therefore the dye-stain demarcation line was drawn shallow to the light pink staining, at the border of red and light pink staining. In previous studies using DD or MVH to detect endpoint differences in PGbased versus PPG-based caries dyes, the light pink staining in the PG group was sometimes kept in place and sometimes removed prior to surface testing. $8.13,16,17$ In the present study, had the dyestain demarcation line been drawn at the border of non-stained and light pink-stained dentin, $\mathrm{L}-\mathrm{L}$ distances would have been greater in some instances. Further study is indicated to determine the tendency of PPG-based dyes to stain dentin light pink, and then to characterize those areas according to hardness, bacterial load, etc.

There are no long-term clinical trials showing improved clinical outcomes for any dye-based excavation endpoints versus conventionallydetermined endpoints, let alone for any one type of dye solution. In fact, clinical studies comparing outcomes for excavation methods in general are quite limited. In one clinical study of restorations in primary teeth, no differences were found at one year regarding marginal adaptation and secondary caries in the chemo-mechanical excavation group compared to the conventional hand excavation group. ${ }^{20}$ In another study of restorations in primary teeth followed for six months, no difference was detected in the survival rate of restorations in the chemo-mechanical excavation group compared to the conventional bur excavation group. ${ }^{21}$ Factors such as restoration longevity, margin integrity, tooth structure integrity, pulpal health, and secondary caries are all relevant considerations. In this regard, PPG-based dye excavation should also be compared to other excavation methods such as chemo-mechanical, polymer bur, and fluorescence-aided excavation, in vitro and clinically. ${ }^{22-24}$ Until such studies are completed, the optimal endpoint for excavation of carious dentin will remain a matter of debate, based primarily on indirect evidence.

\section{CONCLUSIONS}

Under the experimental conditions and compared to PG-based sulforhodamine B caries dye solution, PPG-based dye solution provides a significantly shallower excavation endpoint by $0.298 \mathrm{~mm}$ 195\% confidence interval $0.240 \mathrm{~mm}$ $0.357 \mathrm{~mm}$ ) in freshly extracted permanent teeth with primary dentin carious lesions. The method is useful for comparing staining endpoints of caries dye formulations.

\section{REFERENCES}

1. Roberson TM. Fundamentals in tooth preparation. In: Roberson TM, Heymann HV, Swift EJ, Sturdevant CM (eds) Sturdevant's Art and Science of Operative Dentistry, St. Louis: Mosby, 2006:310.

2. Sato Y, Fusayama T. Removal of dentin by fuchsin staining. J Dent Res 1976;55:678-683.

3. Fusayama T. Two layers of carious dentin: diagnosis and treatment. Oper Dent 1979;4:63-70.

4. Fusayama T. Clinical guide for removing caries using a caries-detecting solution. Quintessence Int 1988;19:397401.

5. Boston DW, Graver HT. Histological study of an acid red 
caries-disclosing dye. Oper Dent 1989;14:186-192.

6. Anderson MH, Loesche WJ, Charbeneau GT. Bacteriologic study of a basic fuchsin caries-disclosing dye. J Prosthet Dent 1985;54:51-55.

7. Miller MB (ed). Reality. Houston: Reality Publishing Co., $1998 ; 12: 51-56$.

8. Hosoya Y, Taguchi T, Tay FR. Evaluation of a new caries detecting dye for primary and permanent carious dentin. $J$ Dent 2007;35:137-143.

9. Trampel R, Schiller J, Naji L, Stallmach F, Karger J, Arnold K. Self-diffusion of polymers in cartilage as studied by pulsed field gradient NMR. Biophys Chem 2002;97:251-260.

10. Cvetkovic A, Picioreanuc C, Straathof AJ, Krishna R, van der Weilen LA. Relation between pore sizes of protein crystals and anisotropic solute diffusivities. J Am Chem Soc 2005; 127:875-879.

11. Ito K, Oikawa M, Kusunoki M, Yokota K, Tsunekawa M. Dental-caries detecting solution. United States Patent Application US 2005/0207978 A1, published September 22, 2005.

12. Oikawa $M$, Kusunoki $M$, Itoh $K$, Hisamitsu $H$. The experimental caries detector containing polypropylene glycol. J Dent Res 2004;83 (Special Issue A):Abstract \#0757 (www.dentalresearch.org).

13. Kinoshita JI, Shinomiya H, Itoh K, Matsumoto K. Light intensity evaluation of laser-induced fluorescence after caries removal using an experimental caries staining agent. Dent Mater J 2007;26:307-311.

14. Konig K, Flemming G, Hibst R. Laser-induced autofluorescence spectroscopy of dental caries. Cell Mol Biol (Noisy-le-grand) 1998;44:1293-1300.

15. Lussi A, Hibst R, Paulus R. DIAGNOdent: An optical method for caries detection. J Dent Res 2004;83(Special Issue C):8083.

16. Itoh K, Kusonoki M, Oikawa M, Hisamitsu H. Removal of caries dentin according to Caries Check staining. $J$ Dent Res 2006;85 (Special Issue B):Abstract \#0754 (www. dentalresearch.org).

17. Hosoya $Y$, Taguchi T, Arita S. Evaluation of new caries detecting dyes for carious dentin. Int J Paediatr Dent 2007;17 (Supplement 1):57,Abstract \#P0116.

18. Fusayama T. A simple Pain-Free Adhesive Restorative System by Minimal Reduction and Total Etching. Tokyo:Ishiyaku EuroAmerica, Inc., 1993:2.

19. Boston DW, Sauble JE. Evaluation of laser fluorescence for differentiating caries dye-stainable versus caries dye-unstainable dentin in carious lesions. Am J Dent 2005; 18:351-354.

20. Kirzioglu Z, Gurbuz T, Yilmaz Y. Clinical evaluation of chemomechanical and mechanical caries removal: status of the restorations at 3,6,9 and 12 months. Clin Oral Investig 2007;11:69-76

21. Bergman J, Leitao J, Kultje C, Gergmann D, Clode MJ. Removing dentin caries in deciduous teeth with Carisolv: a randomized, controlled, prospective study with six-month follow-up, comparing chemomechanical treatment with drilling. Oral Health Prev Dent 2005;3:105-111.

22. Munshi AK, Hegde AM, Shetty PK. Clinical evaluation of Carisolv in the chemico-mechanical removal of carious dentin. J Clin Pediatr Dent 2001;26:49-54.

23. Silva NR, Carvalho RM, Pegoraro LF, Tay FR, Thompson VP. Evaluation of a self-limiting concept in dentinal caries removal. J Dent Res 2006;85:282-286.

24. Lennon AM. Fluorescence-aided caries excavation (FACE) compared to conventional method. Oper Dent 2003;28:341345. 